\title{
Design of thermal energy storage unit for Compressed Air Energy Storage system
}

\author{
Maciej Szybiak ${ }^{1}$, and Maciej Jaworski ${ }^{1, *}$ \\ ${ }^{1}$ Warsaw University of Technology, Faculty of Power and Aeronautical Engineering, Nowowiejska 24, 00-665 Warsaw, Poland
}

\begin{abstract}
The aim of this paper is to present a new concept of a high-temperature thermal energy storage (TES) for the application in the compressed air energy storage (CAES) systems. The proposed storage unit combines the advantages of pressurized containers with packed beds, e.g. of rocks, with the strengths of non-pressurized systems such as those encountered in CSP plants. Designed TES unit consists of the heat exchanger located inside a high-temperature thermocline-type vessel with molten HITEC ${ }^{\circledR}$ salt used as a heat storing material. In terms of the geometry of the designed heat exchanger, a tube-in-tube helical coil type was chosen due to its higher convective heat transfer coefficients in comparison with straight tubes. To find the most suitable case, four helical coils with different dimensions (diameter, pitch) were considered. Heat transfer and pressure drop analysis for each configuration were conducted. In particular, convective and overall heat transfer coefficients as well as friction factors were computed based on the empirical correlations. To verify the obtained results, the analysis based on numerical approach has been carried out with the use of ANSYS Fluent software for the most suitable case.
\end{abstract}

\section{Introduction}

Nowadays we are facing the necessity of making the transition from energy systems based on fossil fuels to ones operating mainly on renewable energy sources. The most serious challenge in this matter is alleviating the consequences of their intermittency. Energy storage seems to be the only technologically simple solution to these problems. Among many techniques of energy storage Advanced Adiabatic Compressed Air Energy Storage (AA-CAES) is a promising and feasible solution on the system level as it provides as large storage capacities as the most often used Pumped Hydro Storage (PHS). Also, power available during discharge of these storage plants is very high, reaching hundreds of MW.

AA-CAES comprises of 4 main components: compressor stage(s), thermal energy storage (TES), compressed air storage (CAS) and turbine stage(s). As compressors and turbines are mostly off-the-shelf products and the choice for CAS systems is rather limited to underground cavities, the main research area is the development of a high efficient TES.

The basic principle how a CAES system works is fairly simple. In times of electricity surplus, it is used to compress ambient air by an electrically driven compressor. Compressed air is cooled down and stored in a pressurized container or underground cavity, e.g. in an underground cavern. If the electrical energy shall be recovered, the air is released, heated up and expanded in a turbine. The turbine in turn drives an electric generator which supplies electrical energy back to the grid. A simplified diagram of the AA-CAES plant is shown in Fig. $1[1,2]$.

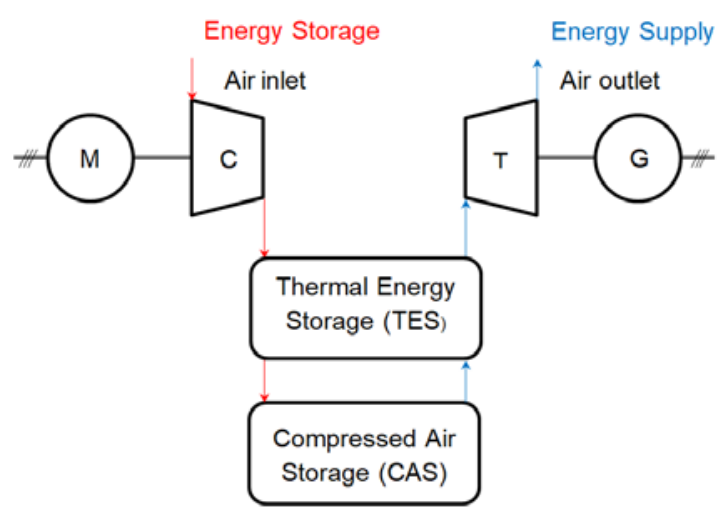

Fig. 1. Simplified diagram of the AA-CAES plant; $\mathrm{M}$ - electric motor driving the compressor, $\mathrm{C}$ - air compressor, $\mathrm{T}$ - turbine, $\mathrm{G}$ - electric generator.

So far, there have been three major CAES concepts: diabatic (D-CAES), (advanced) adiabatic ((A)A-CAES) and isothermal (I-CAES) technologies. All of them differ from one another only in the way how the heat generated during the air-compressing phase is managed. In the case of diabatic CAES, heat is irreversibly lost in the environment. Therefore, an additional source of energy is required to heat the air up before it expands in a turbine. On the other hand, AA-CAES preserves the heat and uses it to raise the temperature of the air during the discharge phase. In the isothermal CAES concept, the air is enabled to constantly exchange heat with the

Corresponding author: mjawo@itc.pw.edu.pl 
environment and so to ensure quasi-isothermal conditions.

The diabatic technology is the simplest one to put into effect but, on the other hand, it requires a significant amount of energy from an additional source. In the vast majority of cases fossil fuels play this role. Dwindling reserves and their contribution to the emission of $\mathrm{CO}_{2}$ and other harmful substances have enhanced the interest for AA-CAES technology, with the TES being the most dynamic field of research. A TES unit designated for an AA-CAES systems must fulfil numerous requirements with the main ones being:

- operating in the temperature range of $50-600^{\circ} \mathrm{C}$,

- stabilizing the outlet air temperature at a given level,

- minimizing heat losses to the environment,

- minimizing air pressure drops.

A number of different high-temperature TES concepts have been proposed and experimentally investigated for the use with air, such as packed beds of rocks or ceramic pieces, large concrete blocks with embedded heat exchangers [3-7]. They remain the most common type of high-temperature TES units. This is because rocks are cheap, easily accessible and they are characterized by a relatively large heat transfer area. However, a pressure vessel must withstand enormous temperature and pressure gradients, and thus, its walls have to be of a significant or even prohibitive thickness. Additionally, loosely stuffed crushed rocks contribute to high pressure drops and periodic thermal stresses, which may lead to attrition of the TES material and, therefore, the necessity of installing air filters.

Another concept of the TES unit, commonly employed in commercial Concentrated Solar Power (CSP) plants, consists of two tanks: one with cold and one with hot oil or molten salt as the heat-transfer fluid (HTF) and thermal energy storage medium [3]. According to [8], especially nitrate salts and their eutectic mixtures are suitable to use as the HTF because of their appropriate thermal properties and no corrosive effects. In addition, they have relatively low melting point, even around $150^{\circ} \mathrm{C}$, and stay stable to over $550^{\circ} \mathrm{C}$. In this temperature range they neither develop toxic gases nor are caustic [8]. A prime example here is the HITEC ${ }^{\circledR}$ salt - an eutectic mixture of potassium nitrate $(53 \%)$, sodium nitrate $(7 \%)$ and sodium nitrite $(40 \%)$ [9]. The main advantages of the two-tank concept are: the pressure part of the heat exchanger is limited only to the air domain, air temperature is kept steady at the outlet, air pressure drops are low. However, such a two-tank system is relatively less compact, requires a greater use of materials and so greater investment costs.

An alternative to the two-tank concept is to store TES medium (hot/cold salt) inside a single tank volume with a separation of the region of different temperatures due to the difference in density of the salt. This concept is called thermocline [10]. In order to increase the thermal capacity of such storage tank inclusion of rocks or macrocapsules containing phase change materials (PCMs) are also considered $[11,12]$. TES unit based on high temperature PCM (eutectic alloy of aluminium, cupper and silicon) for CAES application is described in [7].

The aim of this paper is to present a concept for a high-temperature TES combining the advantages of pressurized packed beds like thermal stratification, with the strengths of non-pressurized systems, in particular those regarding safety issues. The concept of TES unit is not new, similar heat storage unit with steam generator embedded inside molten salt container for the application in CSP is analysed in [13]. In the study reported in this paper the main goal was to check the possibility of developing compact enough heat exchanger that fulfils requirements for cooling/heating of compressed air in a relatively high range of temperature, and that could be enclosed inside heat storage unit. The final effect of the research analysis includes a design of a lab-scale prototype of a thermal energy storage unit for AA-CAES with molten HITEC salt used as a storing material.

\section{Prototype design of the TES unit}

A scheme of the lab-scale prototype of TES unit integrated with heat exchanger is shown in Fig. 2.

A counter-flow, helical coil heat exchanger is used to transfer heat from the air to the molten salt (HITEC). The heat exchanger is located inside a high-temperature thermocline-type thermal energy storage unit with molten salt used as a storing material.

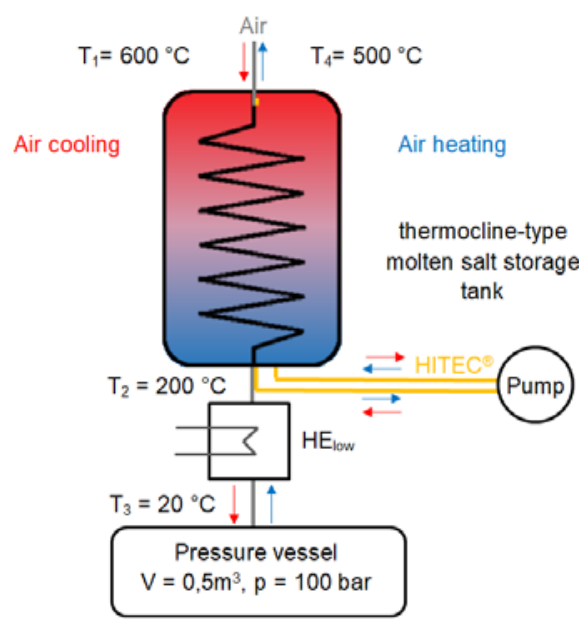

Fig. 2. The lab-scale prototype scheme; $\mathrm{HE}_{\mathrm{low}}$ - low temperature heat exchanger; $T_{1}, T_{2}$ - air temperatures at the inlet and outlet of the TES unit/heat exchanger, $T_{3}$ - final air temperature during charging, $T_{4}$ - final air temperature during discharging.

Compressed hot air flows through a tube (grey line) to the tank filled with molten salt. In the upper part of the tank begins the counter-flow, helical coil tube-intube heat exchanger (black polyline) with the air flowing in the inner tube and molten salt in the outer annulus. At this point, the molten salt may freely flow in and out of the heat exchanger (short yellow line) - the direction depends on whether heat is being absorbed or released. After cooling down to around $200^{\circ} \mathrm{C}$, air leaves the tank and flows through the low temperature heat exchanger 
(e.g. pressurised water - air heat exchanger) to reach the pressure vessel, where it is stored at approximately $20^{\circ} \mathrm{C}$ and pressure 100 bar. The counter-flow of the molten salt shall be provided by an outer pump. The pump shall be connected to the stub of the helical coil heat exchanger as well as directly to the tank's inside (yellow lines).

As for the geometry of the designed heat exchanger, a tube-in-tube helical coil type was chosen due to its higher convective heat transfer coefficients in comparison with straight tubes. To find the most suitable case, four helical coils with different dimensions (diameter, pitch) were considered - heat exchangers available on the market were taken into account. Their dimensions and a scheme are shown in Table 1 and in Fig. 3.

Table 1. Dimensions of the tubes in the tube-in-tube heat exchanger (in $\mathrm{mm}$ ).

\begin{tabular}{|l|c|c|c|c|c|}
\hline \multicolumn{2}{|l|}{ Configuration \# } & 1 & 2 & 3 & 4 \\
\hline \multirow{2}{*}{ Inner tube (air) } & $d_{i, o}$ & 6.4 & 9.5 & 12.7 & 19.1 \\
& $d_{i, i}$ & 4.6 & 7.1 & 9.3 & 15.7 \\
\hline Outer tube & $d_{o, o}$ & 12.7 & 19.1 & 25.4 & 31.8 \\
(molten salt) & $d_{o, i}$ & 10.9 & 16.7 & 22 & 28.4 \\
\hline Coil diameter & $D$ & 101,6 & 152,8 & 203,2 & 254,4 \\
\hline Pitch & $H$ & 15 & 24 & 30 & 37 \\
\hline
\end{tabular}

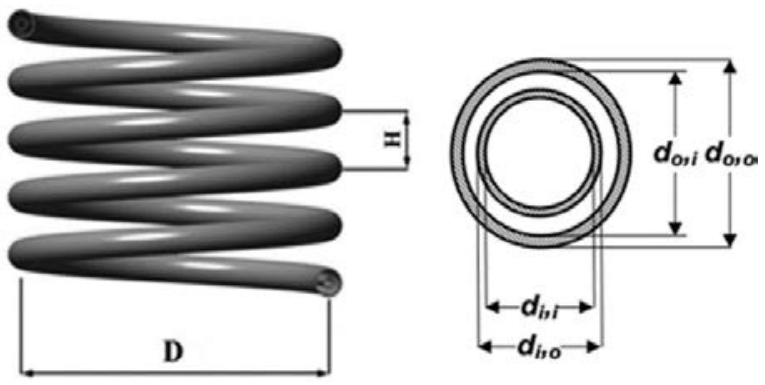

Fig. 3. A scheme of a tube-in-tube helical coil [14].

\section{Heat transfer calculations}

The specific aim of the work was to design a heat exchanger (heat storage unit) of a given internal structure that would ensure the cooling of air by 400 degrees (from $600^{\circ} \mathrm{C}$ to $200^{\circ} \mathrm{C}$ ), while warming up the HITEC salt from $160^{\circ} \mathrm{C}$ to $530^{\circ} \mathrm{C}$. With the assumed mass flow rate of air $(0.01 \mathrm{~kg} / \mathrm{s})$ and, thus, resulting from a simple heat balance calculations mass flow rate of the storage medium $(0.0074 \mathrm{~kg} / \mathrm{s})$, the size of heat exchanger is the main result of the analysis.

The first step in the heat transfer analysis included identification of the thermo-physical properties of air, HITEC salt and stainless steel $304 \mathrm{H}$ in the range 150$600^{\circ} \mathrm{C}$. Although these were easily accessible for HITEC salt [15] and stainless steel $304 \mathrm{H}$ [16], that was not the case for air because the vast majority of sources provide information for pressure $p_{a}=1 \mathrm{bar}$. As it was reported by [17], a relative error caused by applying an ideal gas model and its properties for air instead of exact values is less than $5 \%$. Thus, all of the air properties at pressure $p$
$=100$ bar were calculated on the basis of the ideal gas model.

All the calculations were carried out using empirical correlations. These in turn require parameters' values at the given temperatures. In such a wide temperature range it is unacceptable to employ single values at e.g. $350^{\circ} \mathrm{C}$ (arithmetic average) as it would lead to some serious errors. Thus, in order to enhance the accuracy of calculations, the whole heat exchanger was divided into 20 sections - with the assumption that air temperature drops by $20^{\circ} \mathrm{C}$ in each section. Although inlet and outlet temperatures at the air side are fixed, these still have to be determined for the HITEC salt side. Here, a simple law of energy conservation for an isolated system was employed:

$$
T_{H, \text { in }, i}=T_{H, o u t, i}-\frac{\dot{m}_{a} C_{a, i}\left(T_{a, i n, i}-T_{a, o u t, i}\right)}{\dot{m}_{H} C_{H, i}}
$$

In addition, log mean temperature difference was computed:

$$
\Delta T_{l n, i}=\frac{\left(T_{a, \text { in }, i}-T_{H, \text { out }, i}\right)-\left(T_{a, o u t, i}-T_{H, \text { in }, i}\right)}{\ln \left(\frac{T_{a, \text { in }, i}-T_{H, \text { out }, i}}{T_{a, \text { out }, i}{ }^{-T} T_{H, i n, i}}\right)}
$$

where: $T_{\text {out }, i}-$ outlet temperature at the $i$-th section, ${ }^{\circ} \mathrm{C}$; $T_{i n, i}$ - inlet temperature at the $i$-th section, ${ }^{\circ} \mathrm{C} ; \dot{m}$ - mass flow rate, $\dot{m}_{a}=0,01 \mathrm{~kg} / \mathrm{s} ; \dot{m}_{H}=0,0074 \mathrm{~kg} / \mathrm{s} ; C_{i}-$ specific heat for an average temperature of the fluid in the $i$-th section, $\mathrm{J} /(\mathrm{kg} \cdot \mathrm{K})$. It was assumed that $T_{a, i n, 1}=$ $600^{\circ} \mathrm{C}$ and $T_{H, o u t, 1}=530^{\circ} \mathrm{C}$ as the maximum suggested operating temperature for air and for the HITEC salt [15]. Indices ' $a$ ' and ' $\mathrm{H}$ ' refer to the air and HITEC salt, respectively.

For each section a computational analysis was performed which included sequential calculation of:

- characteristic dimensionless numbers,

- convective heat transfer coefficients,

- heat transfer coefficients related to the unit length,

- required lengths of the sections,

- overall heat transfer coefficients.

Based on the value of Reynolds number, Nusselt number for every section was calculated using one of the two following empirical correlations $[18,19]$ :

- turbulent flow, for $10^{4}<\operatorname{Re}<5 \cdot 10^{6}$ and $0.6<\operatorname{Pr}<$ 2500:

$$
N u=0,021 R e_{f}^{0,8} P r_{f}^{0,43}\left(\frac{P r_{f}}{P r_{w}}\right)^{0,25} \varepsilon_{L}
$$

- laminar flow, for $\operatorname{Re}<2000$ :

$$
N u=0,15 R e_{f}^{0,33} P r_{f}^{0,43} G r_{f}^{0,1}\left(\frac{P r_{f}}{P r_{w}}\right)^{0,25} \varepsilon_{L}
$$

where: $\varepsilon_{L}$ - correction factor for $L / d<50 ; L$ - length of the tube, $d$ - inner diameter.

The correlation for turbulent flow was used for air flow, where Reynolds numbers were in the range from 22,000 to 105,000 , depending on the configuration and the position of the section in the coil. The correlation for laminar flow was used for molten salt flowing in the 
annulus gap - Reynolds number varied in the range from 40 to 1140 .

For the curved conduits correlation (3) or (4) must be multiplied by a correction factor $\varepsilon_{R}[10]$ :

$$
\varepsilon_{R}=1+1,77 \frac{d}{R}
$$

where: $R$ - radius of curvature, $d$ - conduit's inner diameter.

For the characteristic numbers with index ' $f$ ' the value of arithmetic mean of inlet and outlet temperatures was used in the determination of the physical properties, whereas index ' $w$ ' stands for a wall temperature as a reference.

Convective heat transfer coefficient for the $i$-th section, $\alpha_{i}$, was computed from the formula:

$$
\alpha_{i}=\frac{N u_{i} \lambda_{i}}{d_{h}}
$$

Heat transfer coefficient related to the unit length for the $i$-th section, $k_{L, i}$, was computed from the formula [18]:

$$
k_{L, i}=\left[\frac{1}{\pi}\left(\frac{1}{\alpha_{i} d_{i, i}}+\frac{\ln \left(d_{i, o} / d_{i, i}\right)}{2 \lambda}+\frac{1}{\alpha_{o} d_{i, o}}\right)\right]^{-1}
$$

where $\alpha_{i}$ and $\alpha_{o}$ represent convective heat transfer coefficients at the inside and the outside of the inner tube; $\lambda$ stands for thermal conductivity of the wall material.

The required length of the $i$-th section, $L_{i}$, was calculated from the formula [18]:

$$
L_{i}=\frac{\dot{Q}}{\Delta T_{l n, i} k_{L, i}}=\frac{\dot{m}_{a} C_{a, i}\left(T_{a, i n, i}-T_{a, o u t, i}\right)}{\Delta T_{l n, i} k_{L, i}}
$$

Overall heat transfer coefficient related to the outer surface of the inner tube for the $i$-th section, $U_{i}$, was computed from the formula [18]:

$$
U_{i}=\frac{\dot{Q}}{\Delta T_{l n, i} \pi d_{i, o} L_{i}}=\frac{\dot{m}_{a} C_{a, i}\left(T_{a, i n, i}-T_{a, o u t, i}\right)}{\Delta T_{l n, i} \pi d_{i, o} L_{i}}
$$

Results of the calculations are listed in Table 2, where arithmetic mean of the values of heat transfer coefficients related to the unit length $\left(k_{L, a v g}\right)$ and overall heat transfer coefficients related to the outer surface of the inner tube $\left(U_{a v g}\right)$ are given. Total length of the coil and minimum height of the helical coil for each case are the most important results of the analysis since they determine the size of the heat exchanger. Variations of an overall heat transfer coefficient along the pipe, in subsequent sections are shown in Fig. 4. The results presented in the table and in the Figure show an interesting relation between diameter of the coil and an overall heat transfer coefficient. With increasing of the diameter both $k_{\text {L.avg }}$ and $U_{\text {avg }}$ decrease due to the decrease of convective heat transfer coeffi-cients, which is associated with decreasing of the veocity of the fluids. However, for the largest coil considered this trend has changed, which can be explained by non-line-arity of the relations of heat transfer coefficients and surface area as the functions of the coil diamater (as in the case of critical radius of insulation [18]).

Table 2. Arithmetic mean of the values of the heat transfer coefficients related to the unit length $\left(k_{L, a v g}\right)$ and overall heat transfer coefficients related to the outer surface of the inner tube $\left(U_{\text {avg }}\right)$.

\begin{tabular}{|l|c|c|c|c|}
\hline Configuration \# & 1 & 2 & 3 & 4 \\
\hline$k_{L, a v g .} \mathrm{W} /(\mathrm{m} \cdot \mathrm{K})$ & 6.38 & 5.55 & 5.29 & 5.56 \\
\hline$U_{\text {avg. }} \mathrm{W} /\left(\mathrm{m}^{2} \cdot \mathrm{K}\right)$ & 317.31 & 276.20 & 263.07 & 276.50 \\
\hline Total length, $\mathrm{m}$ & 12.61 & 14.50 & 15.22 & 14.50 \\
\hline $\begin{array}{l}\text { Min. height of the } \\
\text { helical coil, } \mathrm{m}\end{array}$ & 0.59 & 0.72 & 0.71 & 0.67 \\
\hline
\end{tabular}

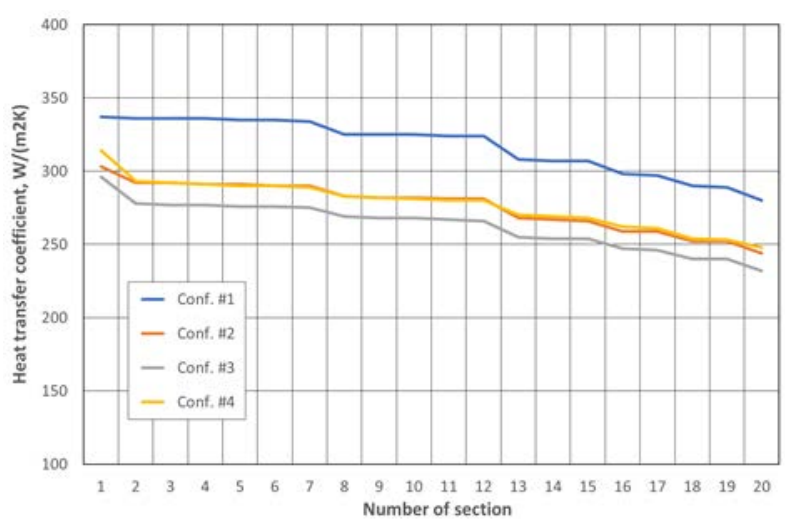

Fig. 4. Overall heat transfer coefficient in subsequent sections.

\section{Pressure drop calculations}

To choose the most suitable dimensions of the tubes an additional pressure drop analysis was conducted as it affects directly performance of the components following the TES, e.g. a turbine. The first step involved deter-mination of the friction factor for each of the 20 sections. Here, a formula proposed in [20] was used:

$$
f=\frac{0.316}{R e^{0.25}}+0.03 \sqrt{\frac{d_{i, i}}{D}}
$$

Subsequently, pressure drop at the $i$-th section, $\Delta p_{i}$, was calculated according to the formula (11) given by [18]. Table 3 illustrates the results of the calculations. Percentage pressure drop includes both phases of the operation, i.e. charging and discharging of TES unit.

$$
\Delta p_{i}=f_{i} \frac{L_{i}}{d_{i, i}} \frac{\rho_{i} v_{i}^{2}}{2}
$$

Table 3. Total and percentage pressure drop related to the air inlet pressure equal to 100 bar.

\begin{tabular}{|c|c|c|c|c|}
\hline Configuration \# & 1 & 2 & 3 & 4 \\
\hline $\begin{array}{c}\text { Total pressure drop, } \\
\mathrm{kPa}\end{array}$ & 230.05 & 32.84 & 9.40 & 0.80 \\
\hline$\%$ & 4.6 & 0.66 & 0.19 & 0.02 \\
\hline
\end{tabular}




\section{Results and discussion}

It may be observed that the total heat transfer coefficient decreased slightly with growing dimensions of the tubes (configuration 1 to 4). However, in each of the cases it stayed at the same level of approximately $300 \mathrm{~W} /\left(\mathrm{m}^{2} \cdot \mathrm{K}\right)$ in the first sections to decline below this value afterwards (Fig. 4). Hence, a simple heat transfer analysis did not determine the most suitable configuration.

On the other hand, differences in the results of pressure drop calculation are much more distinct. There is a clear tendency for the pressure drop to decrease as the inner diameter increases which is in agreement with the theory yielding $\Delta p_{i} \sim d^{-5}$ while keeping mass flow rate unchanged. Total pressure drop in the whole cycle charging/discharging related to the air inlet pressure equal to 100 bar experienced a significant decline along with dimensions growth. Starting at around $4.6 \%$ for the first configuration, it dropped to approximately $0.02 \%$ for the configuration \#4. Keeping in mind that the overall heat transfer coefficients were roughly at the same level, configuration \#4 was found the most suitable and thus, it was subjected to further analysis.

\section{Numerical simulations of heat transfer and fluid flow in the heat exchanger}

To verify the obtained results, an analysis based on numerical approach has been conducted with the use of ANSYS Fluent software.

To assure adequate accuracy of calculation, the computational domain for heat transfer and pressure drop analysis spreads only for the first two sections with the total length of $1080 \mathrm{~mm}$. Grid system for the coils is shown in Fig. 5. It was composed of 453768 elements and 468318 nodes with max. element size of $1 \mathrm{~mm}$ within the boundary layer at the inner (air) side.

Solver type was set to pressure-based as within the analysed domain air density was assumed to be constant. Similar approach was adopted towards the rest of parameters of air, HITEC salt and AISI 304H stainless steel. Their values are listed in Table 4.

Table 4. Properties of air, HITEC salt and AISI 304H stainless steel used in numerical computation.

\begin{tabular}{|l|c|c|c|}
\hline Parameter & Air & HITEC & $\begin{array}{c}\text { AISI } \\
\text { 304H ss }\end{array}$ \\
\hline Density, $\mathrm{kg} / \mathrm{m}^{3}$ & 40.83 & 1740 & 8030 \\
\hline Specific heat, $\mathrm{J} / \mathrm{kg} \cdot \mathrm{K})$ & 1109 & 1567 & 500 \\
\hline $\begin{array}{l}\text { Thermal conductivity, } \\
\mathrm{W} /(\mathrm{m} \cdot \mathrm{K})\end{array}$ & 0.06 & 0.571 & 20 \\
\hline Dynamic viscosity, $\mathrm{Pa} \cdot \mathrm{s}$ & $3.851 \cdot 10^{-5}$ & $1.3 \cdot 10^{-3}$ & \\
\hline Inlet temperature, ${ }^{\circ} \mathrm{C}$ & 600 & 492 & \\
\hline Mass flow rate, $\mathrm{kg} / \mathrm{s}$ & 0.01 & 0.0074 & \\
\hline
\end{tabular}

The realizable $k-\varepsilon$ model of turbulence with enhanced wall treatment was adopted to simulate turbulent flow. The boundary conditions were set as follows: inlet temperature: air $-873 \mathrm{~K}$, HITEC salt $-765 \mathrm{~K}$; mass flow rate: air $-0.01 \mathrm{~kg} / \mathrm{s}$, HITEC salt $-0.0074 \mathrm{~kg} / \mathrm{s}$; outer wall heat flux $-0 \mathrm{~W} / \mathrm{m}^{2}$. To solve pressure-velocity coupling the SIMPLEC scheme was used as well as the second-order upwind scheme for the convection terms in the governing equations. Results of the simulation are shown as contours in Fig. 6 (inner tube, air) and Fig. 7 (annulus, HITEC salt).

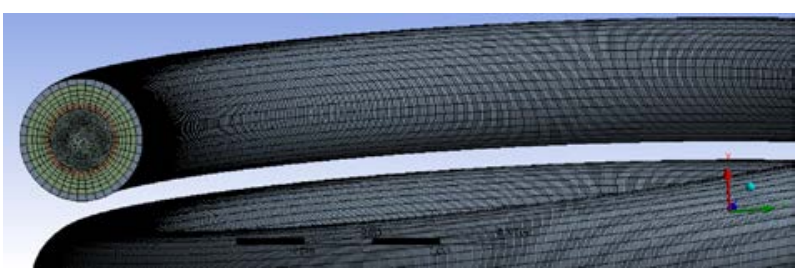

Fig. 5. Grid system for helical coil tube-in-tube heat exchanger.

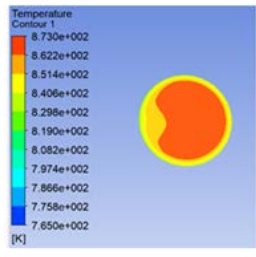

$90^{\circ}$

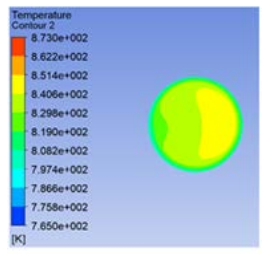

$360^{\circ}$

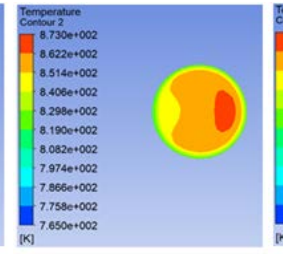

$180^{\circ}$

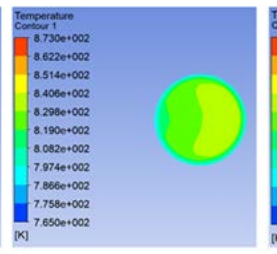

$450^{\circ}$

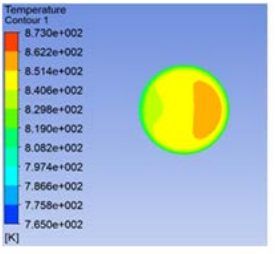

$270^{\circ}$

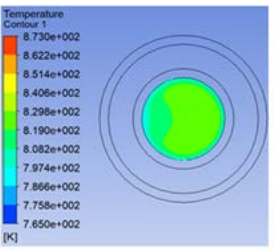

Outlet (air)
Fig. 6. Air temperature fields for inner tube; angle value is measured from the air inlet cross-section.

As it is depicted in Fig. 6, air outlet temperature is in the range of $819-829 \mathrm{~K}$. Comparing these values to the one obtained by means of empirical correlations, that is $833 \mathrm{~K}$, a slight discrepancy may be observed of roughly 4-14 $\mathrm{K}$. The annulus region is characterised by larger differences in results as in the outlet (salt) temperature in a great part of the cross-section is in the range of 808$819 \mathrm{~K}$. On the other hand, a significant part stays in the temperature area of 797-808 K. Here, the temperature obtained by empirical correlation was $803 \mathrm{~K}$.

Concluding from Fig. 6 and Fig. 7, a clear influence of centrifugal force on the temperature distribution may be observed. As a result, secondary flows are induced and particles are forced to move from the inner to the outer part of the tube. Consequently, these radial flows cause more turbulence and so enhance heat transfer. The effects of centrifugal force are particulary visible in the inner region because of much higher velocities of air in comparison to those encountered in the annulus (approximately $1.3 \mathrm{~m} / \mathrm{s}$ to $0.01 \mathrm{~m} / \mathrm{s}$ ).

A similar analysis was conducted for pressure drop in the first 2 sections. The results are depicted in Fig. 8. As it may be seen, pressure drop over these parts of the heat exchanger is in the range of 77-88 $\mathrm{Pa}$. For comparison, 
formula (11) yielded $80 \mathrm{~Pa}$. Thus, a relative error is of the order of a few per cents.

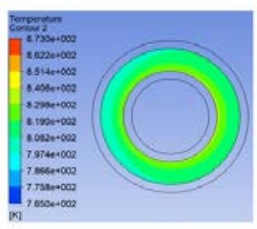

Outlet (salt)

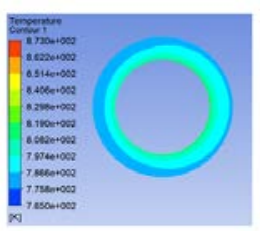

$270^{\circ}$
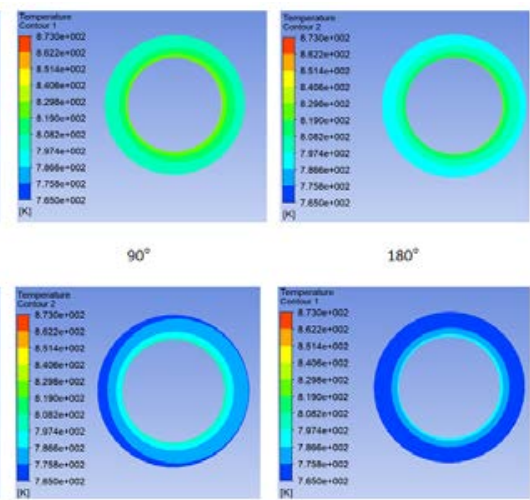

$360^{\circ}$ $180^{\circ}$

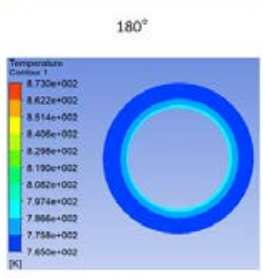

$450^{4}$
Fig. 7. HITEC salt temperature fields; angle value is measured from the air inlet cross-section.

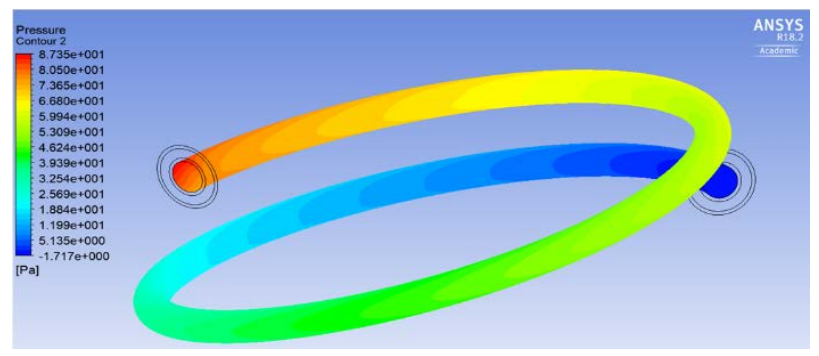

Fig. 8. Pressure drop over the first 2 sections expressed as an overpressure required to reach $0 \mathrm{~Pa}$ at the outlet cross-section.

\section{Summary and conclusions}

The final effect of the analysis performed in the study includes a design of a lab-scale prototype of a $17 \mathrm{kWh}_{\mathrm{th}}$ thermal energy storage unit for AA-CAES with molten HITEC salt used as a storing material and AISI 304H stainless steel as a wall material. Designed TES unit consists of a tube-in-tube helical coil heat exchanger, with tubes diameters 15.7, 19.1, 28.4, 31.8 and coil radius 127.2 , pitch 37 , height 670 and total length 14500 (values in $\mathrm{mm}$ ), located inside a high-temperature thermocline-type vessel with molten HITEC salt used as a storing material. The outer diameter and height of the vessel were set to be 400 and $800 \mathrm{~mm}$, respectively. Air inlet pressure is equal to 100 bar. Its temperature drops from $600^{\circ} \mathrm{C}$ to $200^{\circ} \mathrm{C}$ while charging of the TES unit. On the other hand, HITEC temperature rises by approx. $370^{\circ} \mathrm{C}$ to reach $530^{\circ} \mathrm{C}$ at the outlet, operating at the pressure of 1.5 bar.

Considering the amount of salt contained only in the area surrounding the heat exchanger (around $106 \mathrm{~kg}$ ), the capacity of the TES unit was estimated to be approx. 61.4 MJ/17 kWh. Given the salt mass flow rate of $0.0074 \mathrm{~kg} / \mathrm{s}$, the heat exchanger may operate for nearly 4 $\mathrm{h}$ at its design point. The proposed height and diameter of the vessel are the minimum ones to enclose the heat exchanger. However, by extending the dimensions of the vessel, it is possible to store more salt and so to lengthen the operational time while keeping the inner part, i.e. heat exchanger, unchanged. Such a flexibility broadens significantly the area of potential application.

It was proved that it is possible to obtain an effective cooling of compressed air in a CAES system by heat storage media using simple unit consisting of heat storage tank integrated with a heat exchanger.

\section{References}

1. M. Budt, D. Wolf, R. Span, J. Yan, Appl. Energ., 170, 250 (2016).

2. M. Minutillo, L.A. Lubrano, E. Jannelli, J Energ. Storage, 4, 135 (2015).

3. H. Xue, A. White, J. Energy Storage, 18, 349 (2018).

4. G. Zanganeh, R. Khanna, C. Walser, A. Pedretti, A. Haselbacher, A. Steinfeld, Sol. Energy, 114, 77 (2015)

5. G. Zanganeh, M. Commerford, A. Haselbacher, A. Pedretti, A. Steinfeld, App. Therm. Eng. 70, 316 (2014).

6. L. Geissbühler et al., J. Energy Storage, 17, 129 (2018).

7. V. Becattini et al., J. Energy Storage, 17, 140 (2018).

8. A. Ferrara, G. Yenetchi, R. Haslett, R. Kosson, NASA Technical Reports 19800021356 (1977).

9. M.M. Kenisarin, Renew. Sust. Energ. Rev. 14, 955970 (2010).

10. J.E. Pacheco, et al., J. Sol. Energ-T ASME, 124, 153 (2002).

11. Z. Yang, and S. V. Garimella, Appl. Energ., 103, 256 (2013).

12. S.M. Flueckinger, S.V. Garimella, Appl. Energ., 116, 278 (2014).

13. A. Pizzolato et al., Energy, 139, 231 (2017).

14. A.K. Pathak, Y.K. Tembhurne, M. Gangwar, I.J. Cur. Res. Rev. 9, 19, 40 (2017).

15. HITEC ${ }^{\circledR}$ Heat Transfer salt parameters; Retrieved from http://stoppingclimatechange.com (last access August 2018).

16. AISI $304 \mathrm{H}$ stainless steel parameters, Retrieved from https://www.steeleaglemalta.com (last access August 2018).

17. M. Szybiak, BSc Thesis, Warszawa (2018).

18. Y.A. Cengel, A.J. Ghajar, Heat and Mass Transfer. Fundamentals and application. (The McGraw-Hill Co., 2011).

19. R. Laskowski, A. Smyk, A. Rusowicz, A. Grzebielec, Entropy, 18, 12, 444 (2016).

20. P. Mishra, S.N. Gupta, Ind. Eng. Chem. Process Des. Dev. 18, 130 (1979). 